\title{
CONSIDERACIONES ECOLÓGICAS SOBRE UNA POBLACIÓN DE LYCIUM ATHIUM (SOLANACEAE) Y AMPLIACIÓN DE SU ÁREA DE DISTRIBUCIÓN
}

\author{
MARÍA E. FIGUEROA ${ }^{1}$ y ANA MARÍA GIMÉNEZ ${ }^{1}$
}

\begin{abstract}
Resumen: Lycium athium (Solanaceae) ha sido descripta como una especie endémica de la provincia de Formosa con muy pocos ejemplares citados a la fecha. Se cita por primera vez una población nativa para la provincia argentina de Santiago del Estero, lo que amplía su área de distribución y sugiere que podría extenderse a Paraguay. Nuevas colectas son necesarias para conocer su estatus en la naturaleza. Asimismo, se realizan contribuciones sobre las características de la población y de su hábitat. La población fue encontrada en la región del interfluvio de los ríos Dulce-Salado, tanto en sitios de vegetación arbustiva como de bosque. Se realizó un estudio comparativo entre ambos sitios. Para la caracterización de la población se tuvieron en cuenta las siguientes variables: densidad de la población, altura media de los individuos, abundancia y cobertura absolutas y relativas a las leñosas arbustivas, y patrón de distribución espacial. Para caracterizar el hábitat se determinó salinidad y acidez/alcalinidad del suelo y la diversidad de especies leñosas y suculentas de cada comunidad. $L$. athium se encontró en suelos con elevada salinidad tanto en sitios con arbustivas halófilas como en áreas abiertas dentro del bosque, aunque estuvo mejor representada en el arbustal. Presentó un patrón de distribución espacial agregado en ambiente de arbustal y aleatorio en bosque. En ambos casos se presentó como una especie dominante en los estratos arbustivos bajos. La composición y diversidad de especies en ambos sitios fue la esperada para el tipo de ambiente dentro de la región.
\end{abstract}

Palabras clave: Lycium athium, distribución, Santiago del Estero.

Summary: Ecological considerations on a population of Lycium athium (Solanaceae) and extention of its distribution area. $L$. athium has been described as endemic to Formosa province with very few specimens cited to date. Cited for the first time a native population for Santiago del Estero Argentina province, extending its range and suggests that it could be extended to Paraguay. New collections are necessary to know its status in the wild. It also contributions on the characteristics of the population and its habitat are made. The population was found in the interfluve Dulce-Salado Rivers, both shrub vegetation and forest. A comparative study was performed between both sites. Population density, average height of individuals, absolute and relative abundance, cover, and spatial distribution pattern were taken into account. Salinity, acidity / alkalinity of the soil and diversity of woody and succulent species were determined. L. athium was found in soils with high salinity both sites halophytic shrub and open areas within the forest, although it was best represented in the scrub. It presented a spatial distribution pattern added in shrubland and random in forest. In both cases it was presented as a dominant species in the low shrub strata. The composition and diversity of species at both sites was expected for the type of environment within the region. In both cases it was presented as a dominant species in the low shrub strata. The composition and species diversity at both sites was expected for the type of environment in the region.

Key words: Lycium athium, distribution, Santiago del Estero.

1 Instituto de Silvicultura y Manejo de Bosques (INSIMA). Facultad de Ciencias Forestales. Universidad Nacional de Santiago del Estero. Av. Belgrano (s) 1912. 4200 Santiago del Estero. Rep. Argentina. E-mail: mefigueroa@conicet.gov. ar, amig@unse.edu.ar 


\section{INTRODUCCIÓN}

El género Lycium, actualmente el único género perteneciente a la tribu Lycieae Hunz. (Levin et al., 2011), posee 92 especies en la forma de arbustos o pequeños árboles. América y Argentina en particular, son las regiones con mayor riqueza de especies. Habitan en ambientes áridos y semiáridos, con frecuencia son halófilas y la mayoría poseen adaptaciones al xerofitismo (Bernardello, 2013).

Lycium es considerado por los investigadores como un valioso sistema natural para estudios en evolución reproductiva (Levin et al. 2015).

Entre las especies de Lycium solo cuatro se distinguen por poseer un fruto atípico para el género, un nuculanio con dos pirenos uniseminados, siendo que la mayoría de las especies producen bayas con numerosas semillas (Levin et al., 2011; Bernardello, 2013). Levin et al. (2015), en un estudio reciente sobre afinidad evolutiva, han demostrado que tres de estas cuatro especies (L. athium, L. californicum y L. minimum) están estrechamente relacionadas y sugieren que este tipo de fruto evolucionó al menos dos veces, y en la cuarta especie (L. ameghinoi), claramente alejada, evolucionó convergentemente. Es posible que estas tres especies se dispersaran a partir de un ancestro común desde América del Norte hacia América del Sur y luego hacia Galápagos (Levin et al., 2015).

De manera particular se encontró que $L$. athium, según datos filogenéticos, morfológicos y geográficos, es el pariente continental más cercano de L. minimum y que probablemente también sea su progenitor (Levin et al., 2015). A pesar de estos hallazgos los autores sugieren que son necesarios más estudios sobre L. athium para definitivamente comprender estas relaciones, pero que actualmente la disponibilidad de especímenes para examinar es limitada.

L. athium es un endemismo argentino hasta el momento citado para una restringida área en la provincia argentina de Formosa y conocida por unos pocos ejemplares (Bernardello, 2013; Levin et al., 2015). Está catalogada como especie amenazada en la categoría 5 de la Lista Roja Preliminar de las Plantas Endémicas de Argentina, Resolución Nro. 84 Secretaría de Ambiente y Desarrollo Sustentable (PlanEAr), que corresponde a plantas de distribución restringida, con poblaciones escasas sobre las que pueden actuar uno o más factores de amenaza.

L. athium fue descripta y publicada por Bernardello (1986). Se trata de arbustos de 1-1.5m de alto, de hojas sésiles, carnósulas, planas. El carácter más distintivo es su fruto, un nuculanio 1-seminado, poco común en el género (Bernardello, 2013; Levin et al., 2015). Este es el único rasgo que permite diferenciarla de otras especies del género, aunque se la puede reconocer con facilidad en campo. Localmente L. athium es conocida como "khiskapuka" en quechua y "espina colorada" en castellano, dado que presenta una coloración rojiza oscura característica en sus ramas y tallos que la distinguen fácilmente en el monte. Los lugareños expresan que las espinas fuertes y rígidas de esta planta producen heridas muy dolorosas y un fuerte ardor en la piel al hincarse con ellas; además pueden formar matas muy extendidas y cerradas impidiendo el paso del ganado. Aunque es conocido el uso local de los frutos como tintes naturales de otras especies de Lycium, conocidas localmente como "ichiviles", no se reportaron usos particulares para "khiskapuka".

Dada la escasez de referencias sobre la especie, es necesario generar nueva información para actualizar las bases de datos nacionales, y contribuir con los avances en las investigaciones sobre filogenia, distribución y ecología de la especie.

Este trabajo se propone contribuir al conocimiento de Lycium athium mediante la descripción de la población y el hábitat en diferentes tipos de vegetación.

\section{Materiales y Métodos}

\section{Sitio de estudio}

El estudio fue realizado en el extremo sur de la antigua llanura aluvial del río Mailín, en la localidad de Quimili Paso, Departamento Salavina, Santiago del Estero (coordenadas 28 $49^{\prime} 1,76^{\prime \prime}$, $63^{\circ} 9^{\prime} 41,06^{\prime \prime}$ ). Esta región pertenece a la zona ecológica del Interfluvio Juramento-Dulce y sur del Río Dulce, según la clasificación del Estudio Integral de la Región del Parque Chaqueño (Chaco Red Agroforestal, 1999) (Fig. 1). 
El clima de la región es semiárido mesotermal. La temperatura media anual es de $20,8^{\circ} \mathrm{C}$ (Boletta et al., 1992). La temperatura media del mes más cálido llega a $26,9^{\circ} \mathrm{C}$ y la del mes más frío es de $12,6^{\circ} \mathrm{C}$. El período medio libre de heladas alcanza los 265/280 días anuales. La precipitación media anual es de $650 \mathrm{~mm}$, la evapotranspiración media anual es de $1450 \mathrm{~mm}$ y el déficit climático medio anual es de $800 \mathrm{~mm}$ (Sistema de Información Geográfica de Santiago del Estero SigSE 2.0., INTA).

La vegetación pertenece a la eco-región del
Chaco Semiárido (Burkart et al., 1999), con bosques de Aspidosperma quebracho-blanco en las porciones elevadas del terreno, con especies de los géneros Lycium sp., Schinopsis sp., Larrea sp. y Prosopis sp.; y comunidades halófilas de Allenrolfea sp., Stetsonia coryne y Prosopis ruscifolia en las depresiones. Los suelos poseen textura francolimosa, aluviales, en fases salinos y/o alcalinos. En general tienen mediana capacidad de retención de agua. El pH es neutro a ligeramente alcalino (Chaco Red Agroforestal, 1999).

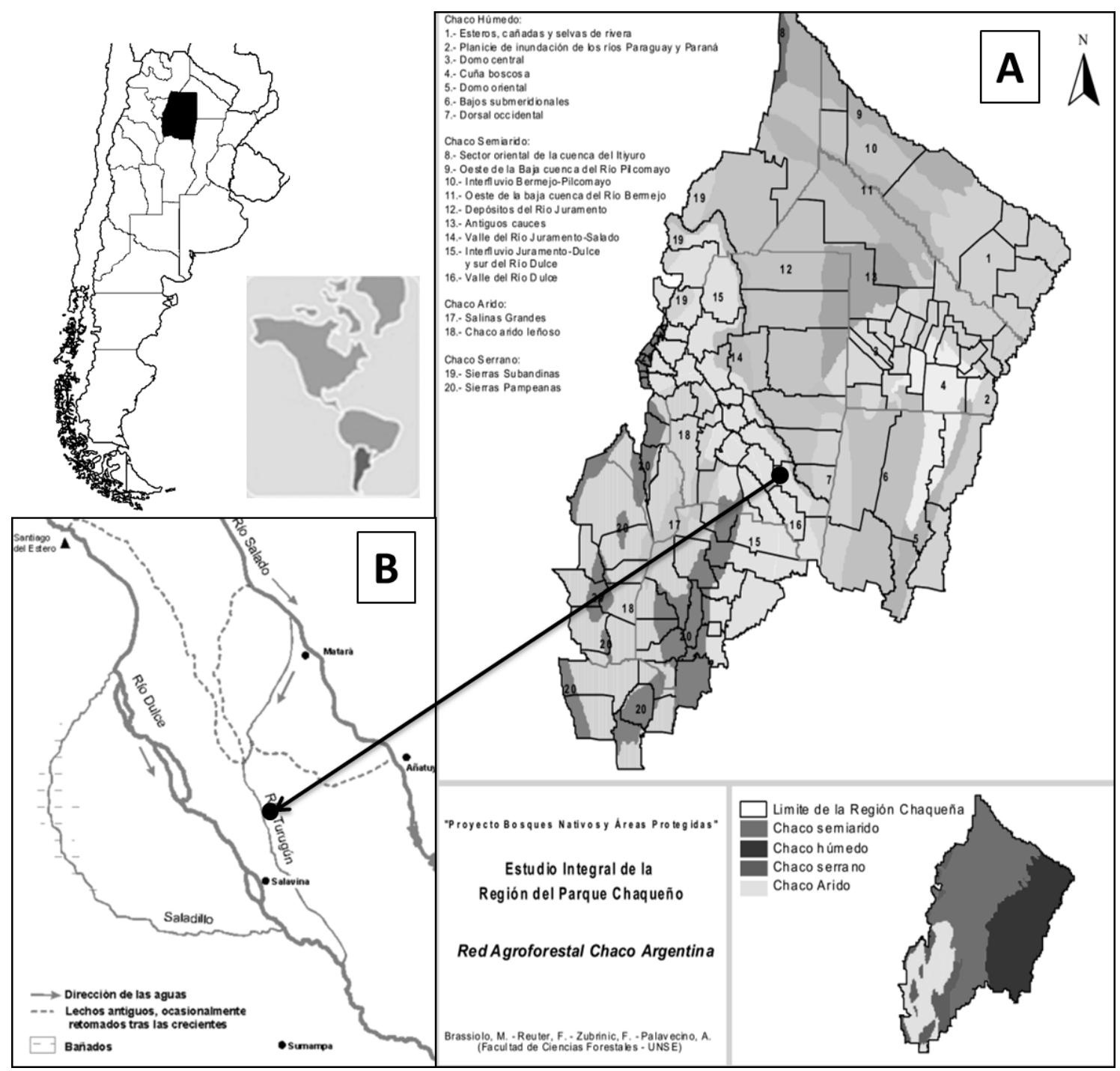

Fig. 1. Ubicación del área de estudio. A: Zonas ecológicas del Parque Chaqueño (1999). B: Río MailínTurungún, años 1901-1974 (Grosso, 2008). 


\section{Material estudiado}

Las muestras de herbario proceden de Santiago del Estero, Dpto. Salaviana, Quimili Paso (Argentina) y fueron depositadas en el Herbario del Museo Botánico Córdoba (CORD). Sus datos de referencia se mencionan a continuación:

Lycium athium Bernardello, G. Bernardello 966.

Lycium americanum Jacq., G. Bernardello 967 у 968 .

Lycium ciliatum Schltdl., G. Bernardello 969 y 970.

Lycium tenuispinosum Miers var. friesii (Dammer) C. L. Hitchc., G. Bernardello 971.

\section{Metodología}

La caracterización de la población de L. athium se realizó mediante las siguientes variables: densidad de la población (ind $/ 100 \mathrm{~m}^{2}$ ), altura media de los individuos, abundancia y cobertura absolutas y relativas a las leñosas arbustivas, y patrón de distribución espacial: agregado, regular o aleatorio. Además se realizaron cortes histológicos del tallo, siguiendo las técnicas indicadas en Zarlavsky (2014), para describir sus principales caracteres anatómicos y detectar rasgos adaptativos y de valor diagnóstico.

Para caracterizar el hábitat se describió el suelo y la diversidad de especies leñosas y suculentas. Los parámetros edáficos utilizados para describir el suelo fueron el grado de salinidad y acidez/alcalinidad de los primeros $50 \mathrm{~cm}$ considerando esta profundidad como límite del área de mayor concentración de raíces dadas las condiciones climáticas y de relieve de la región. Estos parámetros se eligieron teniendo en cuenta que muchas especies de Lycium suelen ser halófilas. Para ello se determinó la conductividad eléctrica $(\mathrm{CE}(\mathrm{dS} / \mathrm{m}))$ y el $\mathrm{pH}$ (en agua) en laboratorio a partir de las muestras de suelo obtenidas en campo en ambientes de arbustal y de bosque. La determinación de la CE se realizó por el método rápido en suspensión suelo: agua de 1:2,5 (dilución de $15 \mathrm{ml}$ de agua destilada y $6 \mathrm{gr}$ de suelo) siguiendo la metodología citada en Lorenz (2005).

Las leñosas se clasificaron en seis categorías según la altura y biotipo: a- arbusto bajo $(<0,6)$; b- arbusto medio $(0,7-1,5)$; c- arbusto alto $(1,6-3)$; d- árbol bajo $(<5)$; e- árbol medio (6-10); f- árbol alto $(>10)$. La identificación y la nomenclatura de las especies se apoyaron en Ragonese (1951) y en el Catálogo de las Plantas Vasculares de Flora de la República Argentina (http://www.floraargentina. edu.ar/).

La colecta de los datos se realizó a lo largo de una transecta de $4 \mathrm{~km}$ de longitud en sentido oeste - este perpendicular a la dirección del cauce atravesando áreas de arbustal y de bosque. Se colocaron 6 parcelas de $4 * 50 \mathrm{~m}$ (3 parcelas en arbustal y 3 en bosque) con su eje mayor orientado en sentido SN. En total la superficie muestreada fue de $1200 \mathrm{~m}^{2}$ (Fig. 2). El muestreo se realizó en verano del 2014. En cada parcela se realizó el conteo del número de individuos de L. athium y de las demás especies leñosas y suculentas presentes. Así mismo se estimó la altura por especie leñosa según las categorías establecidas.

Para caracterizar el patrón de distribución espacial se diseñó una grilla de 50x50m en un sector de arbustal y de bosque. La grilla fue subdividida en celdas de 10x10m en cada sector. En cada celda se contabilizaron los individuos presentes y se estimó el porcentaje de cobertura utilizando la escala de Braun Blanquet (1932).
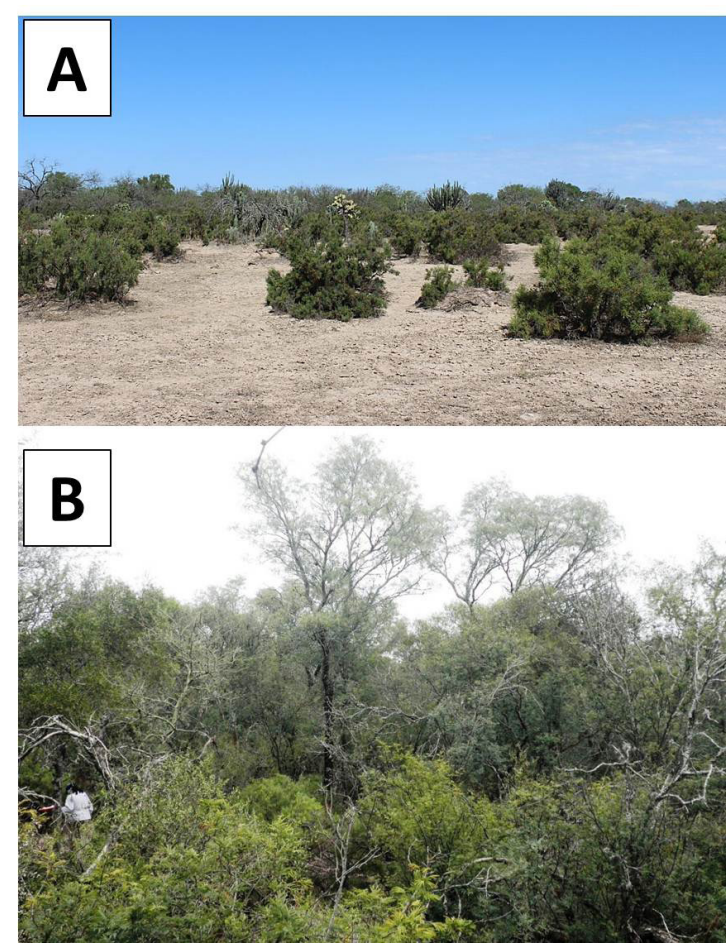

Fig. 2. Tipos de vegetación: A: arbustal de la antigua llanura de inundación del río Mailín. B: bosque abierto de llanura. 
Para determinar la salinidad y acidez/alcalinidad del suelo se tomaron 3 muestras compuestas de suelo de los primeros $20 \mathrm{~cm}$ y de 20 a $50 \mathrm{~cm}$, en 3 puntos tomados al azar en áreas de arbustal y de bosque.

\section{Análisis estadístico}

Las características de la población y el hábitat consideradas en este estudio se compararon según el tipo comunidad: bosque y arbustal. En las comparaciones estadísticas se emplearon pruebas de $\mathrm{T}$ para muestras independientes para un $\mathrm{p}=0,05$ calculadas con el software InfoStat (Di Rienzo et al., 2014). Se calculó el índice de valor de importancia (I.V.I.) con los valores de abundancia y cobertura relativa (Mostacedo \& Fredericksen, 2000).

Para describir el tipo de patrón de distribución se emplearon los datos del número de individuos por celda, provenientes de ambas grillas, y se sometieron a un análisis espacial por índices de distancia (Quero, 2006) mediante el software SADIE Shell v 2.0 (C) 2008 Kelvin F. Conrad). El programa genera un índice de agregación, Ia, que describe el patrón espacial general de los datos: agregado, Ia $>1$, aleatorio $\mathrm{Ia}=1$ y regular $\mathrm{Ia}<1$; y un índice de agrupación ( $v$ ) local en cada posición muestreada. Para ilustrar estos patrones se realizaron mapas con los índices de agrupación local, mediante una interpolación lineal con el software Surfer v 8.01 (Surface Mapping System (C) 1993-2002, Golden Software, Inc).

Los valores de CE se corrigieron en función del volumen de agua en capacidad de campo, para una textura franco limoso y una densidad aparente baja, usando un factor de conversión de 0.39. Para realizar las comparaciones de $\mathrm{CE}$ y $\mathrm{pH}$ entre ambientes, se empleó la media ponderada por la profundidad del horizonte para cada muestra según la ecuación $\mathrm{y}=((\mathrm{H} 0-20 * 2)+(\mathrm{H} 20-50 * 3)) / 5$.

En el caso de la diversidad de especies leñosas y suculentas, los análisis comparativos y descriptivos se realizaron con el software Past versión $2.17 \mathrm{c}$ (Hammer et al. 2001).

\section{Resultados y Discusión}

La nueva cita corresponde a una población encontrada en la región del interfluvio de los ríos
Dulce-Salado en la provincia de Santiago del Estero (Fig. 3). Sin bien no se realizaron estudios fenológicos se pudo advertir en recorridos de campo durante diferentes estaciones del año, que pierde su follaje durante el invierno, cuya estrategia es interrumpir su actividad vegetativa durante el período seco para retomarla durante la estación humedad (verano) cuando el suelo superficial mantiene mayor humedad. Este comportamiento también ha sido reportado para otras especies de Lycium en ecosistemas áridos de Argentina (Villagra et al., 2011, Bucci et al., 2011).

En cortes histológicos del tallo en sección transversal se pudieron observar signos de adaptación al xerofitismo con la presencia de elementos de pequeñas dimensiones (Baas \& Carlquist 1985) (Fig. 4): poros múltiples y solitarios, con tendencia a orientación dendrítica, pequeños (25-37 $\mu \mathrm{m}$ de diámetro tangencial), y poco numerosos; parénquima escaso apotraqueal reticulado o en bandas; fibras abundantes y con paredes medias a gruesas (diámetro externo: $15 \mu \mathrm{m}$, espesor de pared: 3,75 $\mu \mathrm{m}$ ). El diámetro de los miembros de vasos fue considerablemente menor a lo descripto para otras especies de Lycium donde el tamaño es mediano (125-150 $\mu \mathrm{m})$ (Bernardello, 1982). Se requieren estudios que permitan explicar si estas diferencias poseen valor sistemático y permiten diferenciar la especie de otras, o son respuestas fenotípicas asociadas al ambiente. En la estructura secundaria del tallo no se observó periciclo y tampoco fibras en el floema, lo cual concuerda con lo encontrado por Bernardello (1982). Las braquiesclereidas en la peridermis son escasas y en formación (Fig. 4F). En el floema inactivo se observa la formación secundaria de células pétreas en forma aislada. En el corte realizado se observaron dos posibles peridermis (Fig. 4D). Los demás caracteres observados son los usualmente esperados para el género (Metcalfe \& Chalk, 1957).

Los individuos de esta especie se encontraron tanto en el arbustal como en áreas abiertas dentro del bosque. En la Tabla 1 se presenta un resumen en el que se comparan las características de la población y el hábitat de L. athium en ambas comunidades.

Arbustal de la antigua llanura de inundación del rio Mailín

L. athium fue la tercera especie más abundante en relación al resto de especies leñosas y suculentas 


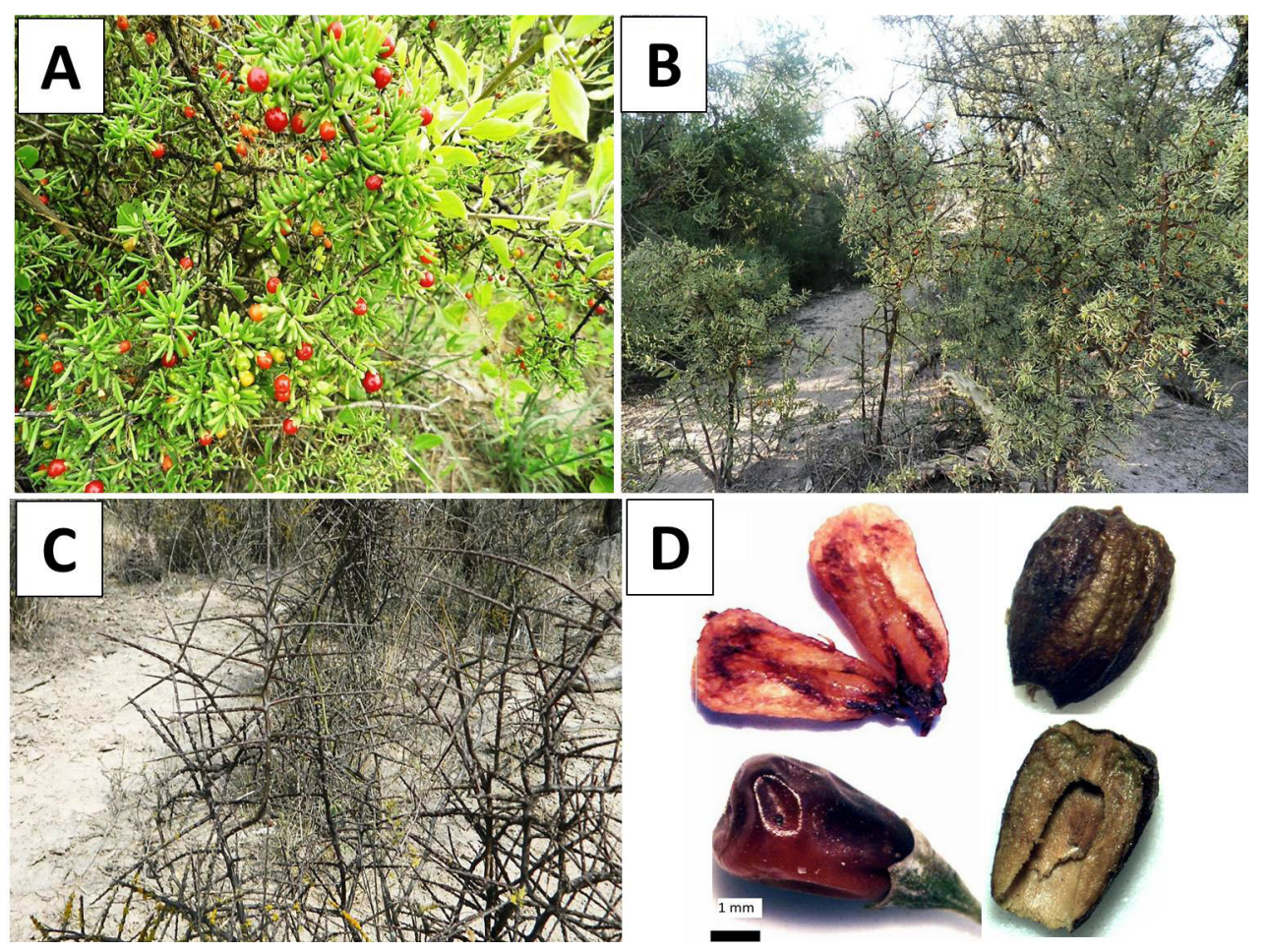

Fig. 3. Lycium athium. A: Detalle de ramas y frutos. B: Aspecto general de tres individuos. C: Ramas sin hojas a finales del invierno. D: Detalle de un fruto con dos pirenos.

censada de la comunidad (12\%). La abundancia relativa al estrato arbustivo fue del $13 \%$. En la categoría de arbustos de mediana altura, predominó con una densidad de $54,5 \mathrm{ind} / 100 \mathrm{~m}^{2}$ y alturas entre $0,30-1,3 \mathrm{~cm}$. Otros arbustos dentro de esta categoría fueron: Lycium americanum, Lycium ciliatum, Lycium tenuispinosum var. friesii y Prosopis sericantha. El porcentaje de cobertura estimado fue de $17,2 \%$, grado 2 en escala de Braun Blanquet (1932). Los individuos presentaron un patrón espacial global agregado según el índice de agregación $(\mathrm{Ia}=1,535, \mathrm{p}$ suba $=0,0064)$. El índice de agrupación local $(v)$ mostró que existen zonas del área de estudio donde el número de individuos se agrupa en manchas (zona con valores altos de la variable) y claros (zona con valores bajos de la variable) (Tabla 1, Fig. 5a).

El suelo mostró elevada salinidad y alcalinidad. La comunidad se compone principalmente por vegetación arbustiva baja $(<2 \mathrm{~m})$ con ejemplares de cactus arborescentes. En general se presenta en ambos márgenes del cauce y en algunos sectores dentro del mismo. La riqueza de especies leñosas fue de 21 ( 6 de árboles y 15 de arbustos) y de suculentas 9. También se registró la presencia de Cyclolepis genistoides, Senna aphylla, Alternanthera nodifera, y arbustos halófilos de Chenopodiaceae: Suaeda divaricata, Atriplex sp, y Heterostachys ritteriana. La especie dominante dentro de los arbustos altos fue Allenrolfea vaginata $(<2 \mathrm{~m})$, sobre la cual generalmente crece el subarbusto apoyante Grahamia bracteata. Entre las especies relevadas, predominaron aquellas asociadas a ambientes salinos, y consideradas en la literatura como halófilas o indicadoras de concentraciones salinas (Ragonese, 1951). Con muy baja densidad se encontraron individuos de Celtis pallida, Capparis atamisquea y Ximenia americana. Entre los arbustos bajos, Lippia salsa, Prosopis reptans y Cortesia cuneifolia fueron los más abundantes. Además se registraron con menor densidad, Aloysia gratissima y la caméfita subfrutescente Talinum polygaloides.

Entre las especies arbóreas se censaron escasos 

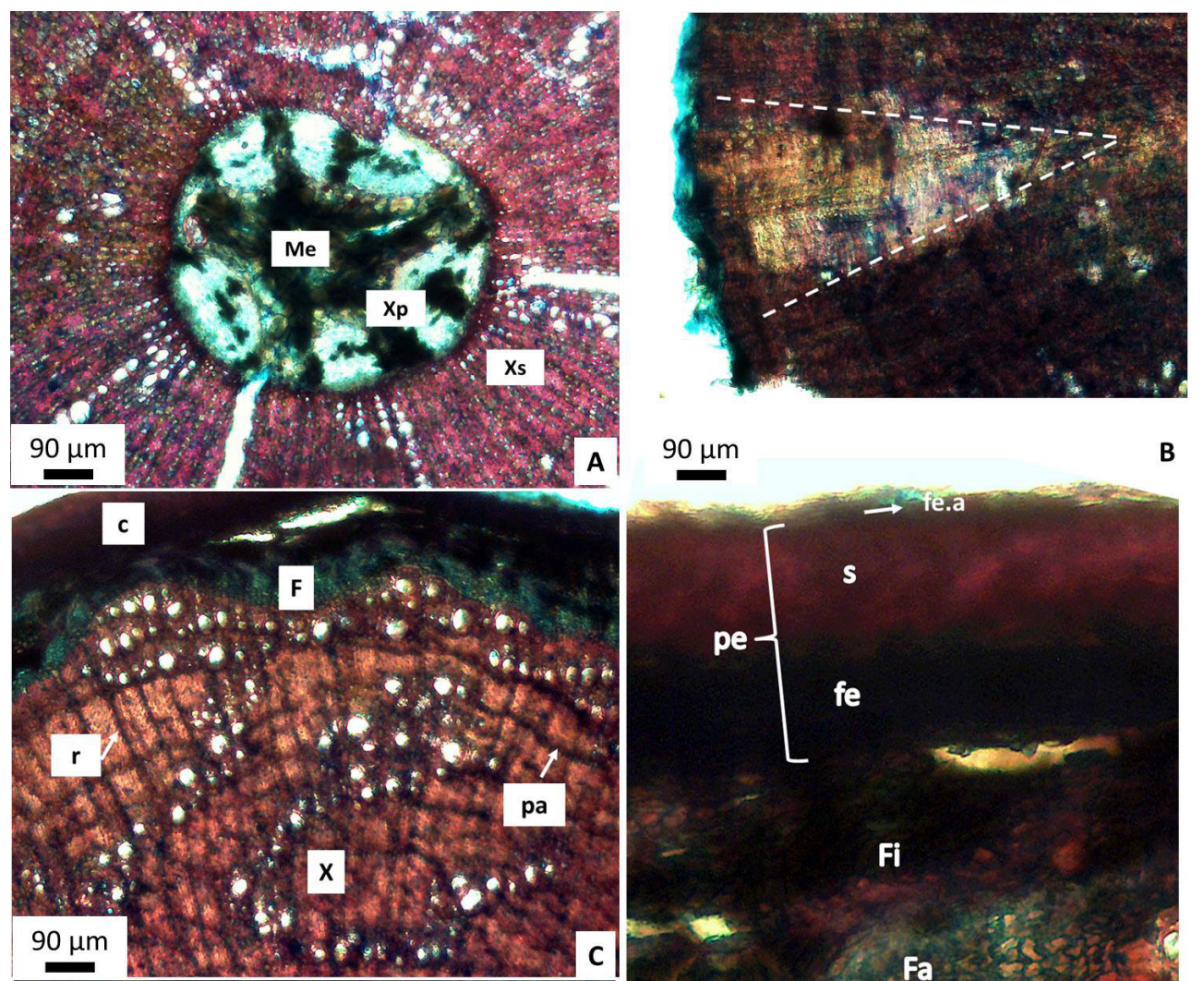

B
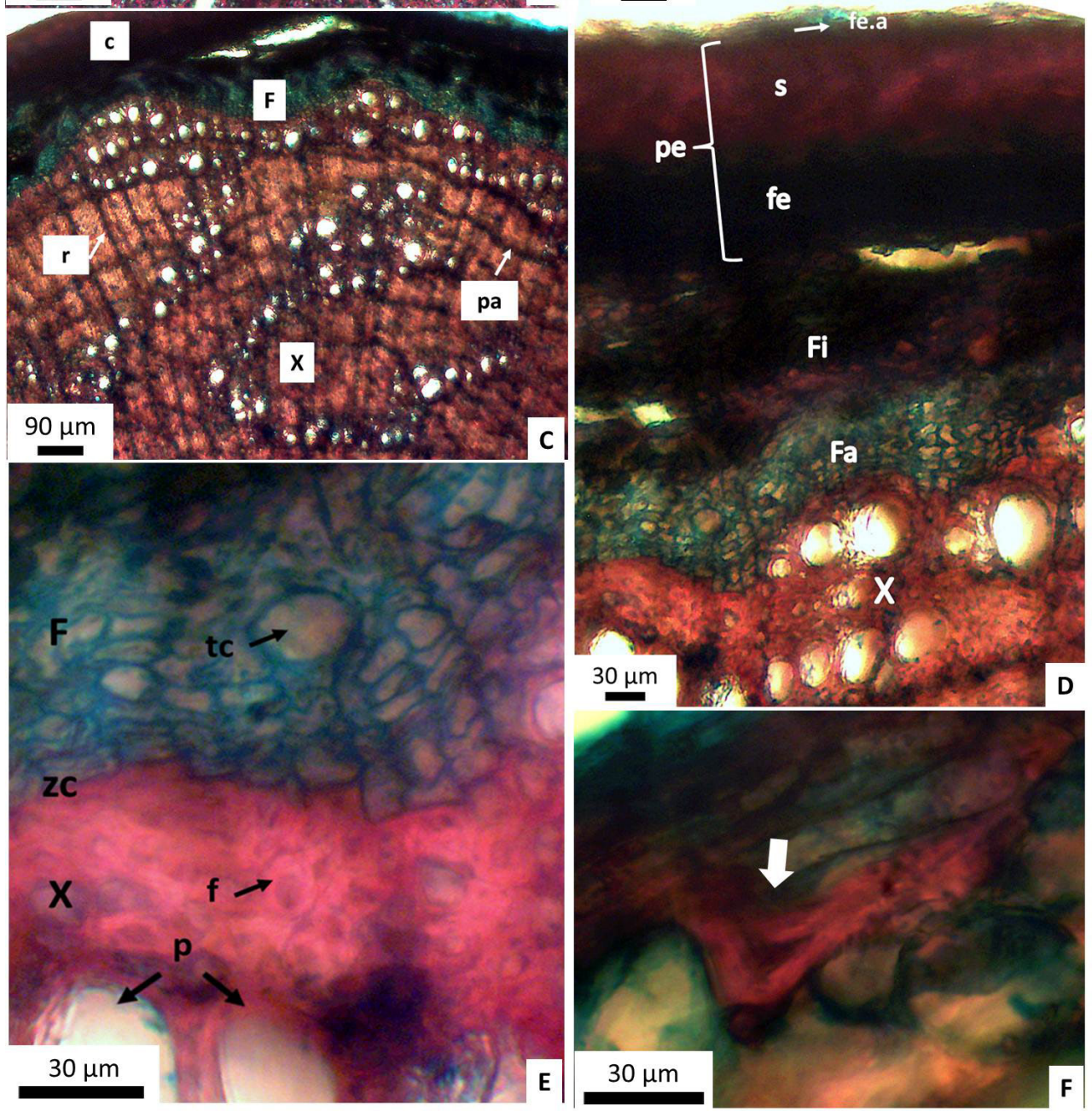

D

Fig. 4. Lycium athium. A: Detalle de la anatomía del tallo, sección transversal. B: ensanchamiento del leño en forma de embudo. C: distribución de los poros. D: detalle de la corteza. E: detalle del xielma y floema. F: detalle de una braquiesclereida en formación. Referencias: Me: médula; Xp: xilema primario; Xs: xilema secundario; pa: parénquima axial; c: corteza; r: radio; F: floema; X: xilema; zc: zona cambial; tc: tubo criboso; p: poro; f: fibra; s: suber; fe: felodermis; Fi: floema inactivo; Fa: floema activo; pe: peridermis; fe.a: felodermis anterior. 
individuos y de bajo porte de Prosopis nigra, Prosopis ruscifolia, Cercidium praecox, Prosopis vinalillo, Geoffroea decorticans y Tabebuia nodosa. Según las características bioecológicas indicadas para estas especies su mayor frecuencia ocurre en suelos salinos (Giménez et al., 2011).

El estrato de suculentas presentó mayor densidad de cactáceas, las de mayor densidad fueron Opuntia anacanta var. kiska-loro (Speg.) R. Kiesling y Opuntia anacanta var. utkilio (Speg.) R. Kiesling, con menor densidad Echinopsis leucantha, Cleistocactus baumannii, Harrisia pomensis, y Opuntia anacanta var. anacanta Speg. También se registraron las arborescentes Stetsonia coryne y Opuntia quimilo, destacándose en el paisaje. Otra especie encontrada fue Bromelia urbaniana.

\section{Bosque abierto de llanura}

L. athium fue el más abundante en el estrato de arbustos de mediano porte $(6,5 \mathrm{ind} / 100 \mathrm{~m} 2)$, con $0,60-1,20 \mathrm{~m}$ de altura, seguido por $L$. ciliatum, Prosopis sericantha y L. americanum con menos de 2 ind $/ 100 \mathrm{~m} 2$, todos ellos ocupando las áreas abiertas dentro del bosque. La abundancia relativa de $L$. athium fue de $6,95 \%$. El porcentaje de cobertura estimado fue de $19,16 \%$, grado 2 en escala de Braun Blanquet (1932). Los individuos mostraron un patrón espacial aleatorio (Tabla 1, Fig. 5b).

El suelo mostró elevada salinidad y alcalinidad. La vegetación del bosque es abierta, discontinua y baja. La altura media del dosel fue de entre 7 y 10 metros y del estrato arbustivo entre 2 y 3 metros. El número de especies arbóreas registradas fue de 8 , arbustivas 16 y de suculentas 3. Las principales especies del dosel fueron Schinopsis lorentzii y Aspidosperma quebracho- blanco. También están presentes: Prosopis nigra, Ziziphus mistol, Geoffroea decorticans, Cercidium praecox, Prosopis ruscifolia. En el estrato arbustivo alto se encontraron: Celtis pallida, Capparis atamisquea, Maytenus vitis-idaea, Allenrolfea vaginata, Grahamia bracteata, Mimosa detinens, Maytenus spinosa, Schinus sp. y Lycium boerhaviaefolium L. f. (ex Grabowskia duplicata). Se destaca en la comunidad Acacia praecox. Entre los arbustos bajos, se encontraron Cortesia cuneifolia y Cyclolepis genistoides y Aloysia gratissima. Entre las suculentas Bromelia urbaniana fue más abundante en tanto que Opuntia salmiana y
Tabla 1: Características de la población y hábitat de $L$. athium en comunidad de bosque y arbustal. Prueba de $T$ para muestras independientes: a- $p=0,0027, b-p=0,724, c-p=0,49, d-p=0,82$ (InfoStat). * Resultados obtenidos con Sadieshell v. 2.0. ${ }^{* *}$ Diferencias significativas Boot $p(e q)<0,05$ (Resultados obtenidos con el programa Past v.

$$
\text { 2.17c). }
$$

\begin{tabular}{|c|c|c|}
\hline Características & Arbustal & Bosque \\
\hline \multicolumn{3}{|l|}{ Población de $L$. athium } \\
\hline Densidad (ind/100m2)- a & 54,5 & 6,5 \\
\hline Abundancia absoluta & 327 & 39 \\
\hline $\begin{array}{l}\text { Ab. Relativa (\%) (Nind L. } \\
\text { athium /Ntotal_arbustos) }\end{array}$ & 13 & 6,95 \\
\hline $\begin{array}{l}\text { Cob. media (\% promedio } \\
\text { por celda de } 100 \mathrm{~m} 2)-\mathrm{b}\end{array}$ & 17,2 & 19,16 \\
\hline Cob. Relativa (\%) & & \\
\hline $\begin{array}{l}\text { (Cob. media L. athium/ } \\
\text { Cob. total_leñosa) }\end{array}$ & 8,8 & 7,6 \\
\hline $\begin{array}{l}\text { I.V.I. (Ab. Relat (\%) } \\
+ \text { cob. Relat. (\%)) }\end{array}$ & 20,8 & 12,05 \\
\hline Altura estimada (m) & $0,30-1,3$ & $0,60-1,20$ \\
\hline \multicolumn{3}{|l|}{ Patrón de distribución* } \\
\hline la & $\begin{array}{c}1,535 \\
\text { agregado }\end{array}$ & $\begin{array}{c}1,01 \\
\text { aleatorio }\end{array}$ \\
\hline P la & 0,0064 & 0,4042 \\
\hline mean v j j & $-1,425$ & $-1,088$ \\
\hline$P($ mean v $\mathrm{j})$ & 0,0127 & 0,2469 \\
\hline mean v_i & 1,340 & 0,821 \\
\hline$P($ mean v_i) & 0,0317 & 0,6114 \\
\hline \multicolumn{3}{|l|}{ Suelo } \\
\hline CE $(\mathrm{dS} / \mathrm{m})$ suelo- $\mathrm{c}$ & 37,43 & 23,96 \\
\hline pH suelo- d & 8,27 & 8,46 \\
\hline \multicolumn{3}{|l|}{$\begin{array}{l}\text { Número de especies } \\
\text { por forma de vida }\end{array}$} \\
\hline Árbol & 6 & 8 \\
\hline Arbusto alto $(1,6-3)$ & 5 & 9 \\
\hline Arbusto medio $(0,7-1,5)$ & 5 & 4 \\
\hline Arbusto bajo $(<0,6)$ & 5 & 3 \\
\hline Suculentas & 9 & 3 \\
\hline \multicolumn{3}{|l|}{ Índices de diversidad } \\
\hline Taxa_S & $30^{* *}$ & $27^{* *}$ \\
\hline Individuals & $2787^{* *}$ & $1189^{* *}$ \\
\hline Shannon_H & $2,197^{\star *}$ & $2,309^{* *}$ \\
\hline Equitability_J & $0.6461^{\star *}$ & $0.7005^{\star *}$ \\
\hline
\end{tabular}




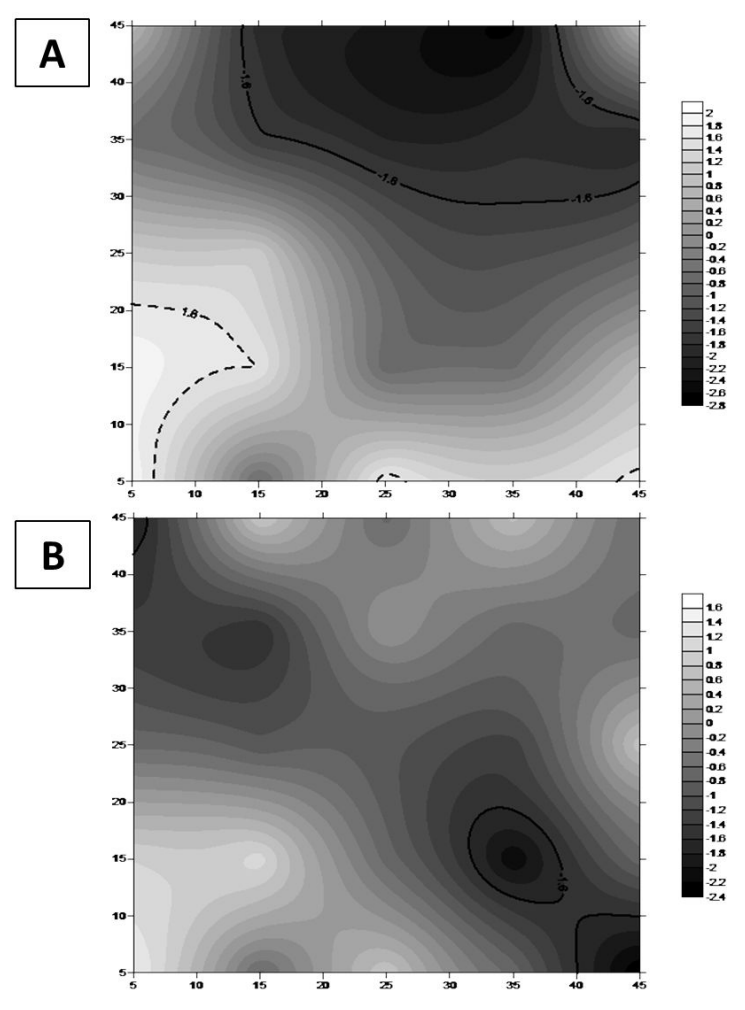

Fig. 5. Mapa de la distribución del índice de agrupación ( $\mathrm{v}$ )en arbustal (A) y bosque (B). Las áreas de color claro indican valores de $v>1,5$ y muestran manchas de agregación (línea discontinua), las áreas oscuras indican valores de $\mathrm{v}<-1,5$ y muestran claros de agregación (línea continua). Se observa una mayor concentración de $L$. athium en las celdas ubicadas hacia la esquina inferior izquierda en A y una densidad significativamente baja en la esquina inferior derecha en $\mathrm{B}$.

Stetsonia coryne presentaron baja densidad. Las características de este bosque concuerdan con las citadas para un bosque bajo o en regeneración según Brassiolo (2005).

\section{Comparaciones entre arbustal y bosque}

L. athium presentó mayor densidad y abundancia de individuos (absoluta y relativa) en el arbustal que en el bosque. No se observaron diferencias en el porcentaje de cobertura estimado, siendo de grado 2 en la escala de Braun Blanquet en ambos casos. La combinación de ambos parámetros en el I.V.I muestra que la especie tiene mayor importancia en el estrato arbustivo del arbustal que del bosque.
El rango de altura de los individuos no varió entre las comunidades. Por lo tanto, esto indicaría que se trata de una especie heliófila que se expresa mejor en áreas abiertas, donde la competencia por luz es menor.

El patrón de distribución agregado que mostró $L$. athium en el arbustal concuerda con la agregación espacial de las plantas que ocurre generalmente bajo condiciones ambientales adversas observado comúnmente en zonas áridas y semiáridas (Tirado \& Pugnaire 2003). Patrones similares fueron encontrados en un matorral desértico micrófilo mexicano, pero estuvieron relacionados al grado de perturbación local de las parcelas, sugiriendo una mayor fragmentación de la población estudiada en las áreas más degradadas (Anaya \& Rubio, 2010). En el mismo estudio se destaca que dentro de las especies arbustivas dominantes, Larrea tridentata, presentó una distribución aleatoria en todas las parcelas, pero formó parches significativos sólo en la parcela más perturbada. En concordancia con este resultado, la agregación que presentó L. athium en el arbustal podría estar relacionada a un ambiente más adverso, donde se observó una mayor salinidad, menor diversidad y equitatividad de especies, y mayor riqueza de cactáceas, consideradas muchas de ellas como indicadoras de degradación según Ragonese \& Castiglione (1970). Así mismo, la agrupación local (v) significativa que se observó en el arbustal, marca dos zonas contrastantes de claros y manchas en la parcela, lo cual estaría indicando la acción de factores que actúan a escala local y pueden estar favoreciendo estas diferencias. Aunque diferentes hipótesis se han formulado para explicar si este patrón se debe más a las condiciones microambientales o la interacción con otras especies (Bochet et al. 1999), muchos estudios en los ecosistemas áridos y semiáridos han asociado la heterogeneidad espacial con la presencia de arbustos e interacciones positivas dentro de la comunidad (Montaña 1992). En este sentido Zúñiga et al., (2005) determinaron que el patrón de agregación espacial encontrado mediante la técnica SADIE para una especie de cactácea de un matorral xerófilo de México, puede ser consecuencia de la relación de nodricismo con ciertas especies arbustivas de la comunidad. $\mathrm{Si}$ bien la distribución global en el bosque fue aleatoria, localmente se observó una zona donde la abundancia de individuos fue significativamente 
menor, lo cual puede deberse a zonas con dosel arbóreo más denso que limitan la presencia de esta especie, lo cual resulta en una menor densidad local de individuos.

Tanto el suelo del arbustal como del bosque presentaron textura franco limoso, elevada conductividad eléctrica y $\mathrm{pH}$. En el arbustal la salinidad fue mayor que en el bosque, pero estas diferencias no fueron estadísticamente significativas. En ambos casos el suelo se clasificó como extremadamente salino $(>12 \mathrm{dS} / \mathrm{m}$ según Velasco Molina (1983) y moderadamente alcalino (Arens \& Etchevehere, 1966). Los valores obtenidos para el bosque resultaron mucho más elevados que los registrados en bosques del chaco árido con influencia salina $(<15 \mathrm{dS} / \mathrm{m}$, Karlin et al., 2012). La elevada salinidad del suelo en el bosque es esperable considerando la presencia de especies indicadoras de suelos salinos (Giménez et al., 2011, Coirini et al., 2010). En general se ha encontrado que muchas las especies chaqueñas poseen un elevado potencial osmótico que les permite superar la presión osmótica del suelo salino (Cercidium praecox: -29; Aspidosperma quebracho-blanco: -30; Schinopsis lorentzii: -30; Ziziphus mistol: -35; Maytenus vitis-idaea: -55; Acacia praecox: -60 (Mitlöhner, 1990). En otros ecosistemas de la región chaqueña se ha citado que las especies de Lycium habitan en bajos salinos y son frecuentes en zonas degradadas (Coirini et al., 2010). También suelen formar parte de comunidades arbustivas casi impenetrables con marcado carácter halófilo junto a Allenrolfea vaginata y mogotes de Cyclolepis genistoides (Lewis et al., 1990; Pire \& Lewis, 1994).

Los índices de riqueza y diversidad de especies leñosas muestran diferencias significativas, siendo el bosque más diverso y equitativo aunque con menor densidad de especies (Taxa_S). L. athium se mantuvo predominante en abundancia en la categoría de arbustos de mediano porte en ambos sitios, aunque la abundancia de individuos leñosos de esta categoría disminuyó en el bosque. El estrato de arbustos bajos estuvo mejor representado en el arbustal que en el bosque (Fig. 6). La composición de especie del estrato arbustivo alto también se vio modificada entre las dos comunidades (Fig. 7). La composición de especies arbóreas, arbustivas y cactáceas coincide con la reportada para los suelos de los planos bajos salinizados descriptos para

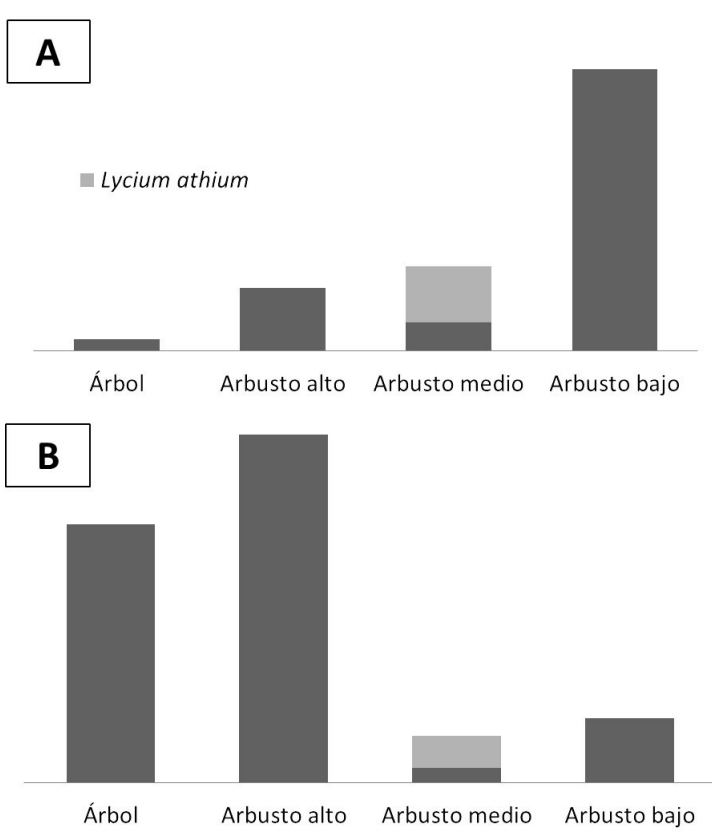

Fig. 6. Abundancia absoluta de individuos por categoría de leñosa en arbustal $(A)$ y bosque (B).

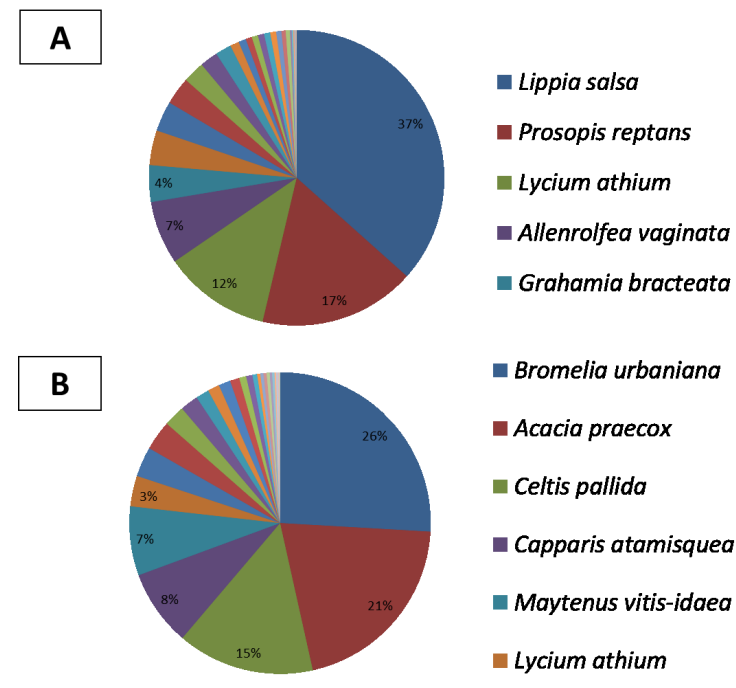

Fig. 7. Composición de especies del arbustal (A) y del bosque (B), expresada en abundancia relativa por especie de leñosa y suculenta. 
la región (Giménez et al., 2007). Los valores de riqueza total y los índices de diversidad (Shannon $\mathrm{H}$ y Equitatividad) del arbustal y del bosque fueron similares a los determinados para los sitos más y menos degradado respectivamente, estudiados por Giménez et al. (2007). Resultados semejantes se encontraron en el estudio de Karlin et al. (2011), donde los ambientes con mayor salinidad presentaron un índice de Shannon promedio de 1,09 y en las zonas de bosque varió entre 3,19 y 3,96, en concordancia con una disminución en la CE. La presencia de mayor densidad de cactáceas en el arbustal podría indicar sobrepastoreo (Ragonese \& Castiglione, 1970).

\section{Conclusiones}

La población de L. athium (espina colorada o khiskapuka), encontrada habita en suelos con elevada salinidad tanto en comunidades arbustivas halófilas como en el bosque xerófilo. En ambos casos fue dominante en los estratos arbustivos bajos. Estuvo mejor representada y con un mayor valor de importancia en el arbustal donde mostró además un patrón de distribución espacial agregado probablemente relacionado a condiciones ambientales adversas. El tallo presentó signos de adaptación al xerofitismo con la presencia de elementos de pequeñas dimensiones. La composición y diversidad de especies en ambos sitios fue la esperada para el tipo de ambiente dentro de la región.

El hallazgo de esta especie en un sitio ubicado a más de $500 \mathrm{~km}$ de distancia del área donde fue encontrada por primera vez, pone de manifiesto que su catalogación como endémica de la provincia de Formosa no corresponde y que es necesario realizar colectas adicionales en otras provincias y en Paraguay para conocer la distribución real de la especie y esclarecer su estatus en la naturaleza. Es probable que el hecho que solo pueda reconocerse con certeza en estado de fruto contribuya a su confusión y que, por ello, no haya sido incluida en los inventarios de biodiversidad realizados hasta la fecha. Este hallazgo incorpora una nueva especie a la flora xero-halófila de la región chaqueña semiárida.

La estrecha afinidad evolutiva entre L. athium de Argentina y L. minimum de Galápagos significa un valioso aporte para comprender el proceso de evolución y adaptación de estas especies. En este sentido L. minimum es el primer caso documentado de sistema sexual dimórfico en una especie de Lycium sudamericana (Levin et al. 2015), lo cual permite inferir que también las poblaciones de L. athium muy probablemente posean el mismo sistema sexual, aunque esto debe ser corroborado con nuevos estudios.

\section{Agradecimientos}

Se agradece la colaboración de Gabriel Bernardello por la identificación de la especie y de Mirta Sposetti por la preparación de los cortes histológicos.

\section{Biblografía}

ANAYA, D. M., \& O. R. G RUBIO. 2010. Análisis espacial por índices de distancia (Sadie) de Lophophora williamsii en tres parcelas con diferente grado de perturbación en San Luis Potosí. Laboratorio de Ecología, Facultad de Ciencias Naturales, Universidad Autónoma de Querétaro.

ARENS, P.L. \& P.H, ETCHEVERE.1966. Normas de Reconocimiento de Suelos. Instituto de Suelos y Agrotecnia (INTA). Tirada interna, Buenos Aires.

BAAS, P \& S CARLQUIST. 1985. A comparison of the ecological wood anatomy of the floras of Southern California and Israel. IAWA Bull. 6:349 -353.

BERNARDELLO, G. 2013. Lycium. En: G. Barboza (ed.), Flora Argentina, volumen 13, Dycotiledoneae, Solanaceae: 47-75. INTA, IMBIV, IBODA, Buenos Aires.

BERNARDELLO L. M. 1982. Estudios en Lycium (Solanaceae). I. Anatomía de hoja y tallo, y sus diferencias con Grabowskia. Bol. Soc. Argent. Bot. 21: 153-185.

BERNARDELLO, L. M. 1986. Revisión taxonómica de las especies sudamericanas de Lycium (Solanaceae) Bol. Acad. Ci. Cordoba 57: 173-356

BERTILLER, M. B., A. J. BISIGATO, A. L CARRERA, \& H. F. DEL VALLE. 2004. Estructura de la vegetación y funcionamiento de los ecosistemas del Monte Chubutense. Bol. Soc. Argent. Bot 39: 139-158.

BOCHET, E., J. L. RUBIO \& J. POESEN.1999. Modified topsoil islands within patchy Mediterranean vegetation in SE Spain. Catena 38: 23-44.

BOLETTA, P.E., L.R. ACUÑA \& M.M. JUAREZ 
DE MOYA. 1992. Análisis de las características climáticas de la provincia de Santiago del Estero y comportamiento del tiempo durante la sequía de la campaña agrícola 1988/89. Informe técnico, INTAUNSE, Santiago del Estero.

BRASSIOLO, M. 2005. Propuestas para la conversión de bosques degradados. Los bisques del Chaco Semiárido. IDIA 2: 23-28.

BRAUN- BLANQUET, J. 1932. Plant sociology; the study of plant comunities. McGraw-Hill, New York.

BUCCI, S. J., F. G. SCHOLZ, P. A. IOGNA \& G.GOLDSTEIN. 2011. Economía del agua de especies arbustivas de las Estepas Patagónicas. Ecología austral 21: 43-60.

BURKART, R., N. O. BÁRBARO, R. O. SÁNCHEZ \& D. A. GÓMEZ. 1999. Ecorregiones de la Argentina. Administración de Parques Nacionales, Buenos Aires.

CATÁlOGO DE LAS PLANTAS VASCULARES DE FLORA DE LA REPÚBLICAARGENTINA. http:// www.floraargentina.edu.ar/ [acceso: 21 de febrero del 2015].

CHACO, RED AGROFORESTAL. 1999. Estudio integral de la región del parque chaqueño. Proyecto Bosques Nativos y Áreas Protegidas Préstamo BIRF (4085-AR).

COIRINI, R.O., M. KARLIN \& G.J. REATI (eds.). 2010. Manejo Sustentable del Ecosistema Salinas Grandes, Chaco Árido. Encuentro Grupo Editor, Córdoba.

DI RIENZO J.A., F. CASANOVES, M.G. BALZARINI, L. GONZALEZ, M. TABLADA \& C.W. ROBLEDO InfoStat versión 2014. Grupo InfoStat, FCA, Universidad Nacional de Córdoba.

GIMÉNEZ, A. M., P. HERNÁNDEZ, R. GEREZ \& N. A. RÍOS. 2007. Diversidad vegetal en siete unidades demostrativas del chaco semiárido argentino. Madera y Bosques 13: 61-78.

GIMENEZ, A., P. M. HERNANDEZ, M. E. FIGUEROA \& I. BARRIONUEVO. 2011. Diversidad del estrato arbóreo en los bosques del Chaco Semiárido. Quebracho (Santiago del Estero) 19: 24-37.

GROSSO, J. L. 2008. Indios Muertos, Negros Invisibles. La Identidad "Santiagueña" en Argentina. Tesis de Doctorado. Departamento de Antropología, Universidad de Brasilia.

HAMMER Ø., D.A.T HARPER, \& P.D. RYAN. 2001. PAST: Paleontological Statistics software package for education and data analysis. Palaeont. Electron. 4: $1-9$.

KARLIN, M. S., E. V. BUFFA, U. O. KARLIN, A. M. CONTRERAS, R. O. COIRINI \& E. R. POSSE. 2012. Relaciones entre propiedades de suelo, comunidades vegetales y receptividad ganadera en ambientes salinos (Salinas Grandes, Catamarca,
Argentina). Revista Latinoam. Recursos Natur. 8: 30-45.

LEVIN, R. A., G. BERNARDELLO, C. WHITING \& J. S. MILLER. 2011. A new generic circumscription in tribe Lycieae (Solanaceae). Taxon 60: 681-690.

LEVIN, R. A., E. M. KEYES \& J. S. MILLER. 2015. Evolutionary relationships, gynodioecy, and polyploidy in the Galápagos Endemic Lycium minimum (Solanaceae). Int. J. Plant Sci. 176: 197-210.

LEWIS, J. P., E. F. PIRE, D. E. PRADO, S. L. STOFELLA, E. A. FRANCESCHI \& N. J. CARNEVALE. 1990. Plant communities and phytogeographical position of a large depression in the Great Chaco, Argentina. Vegetatio 86: 25-38.

LORENZ, G. 2005. Métodos de análisis de suelos. Serie Didáctica $\mathrm{N}^{\mathrm{o}} 12$. Cátedra de edafología forestal, Facultad de Ciencias Forestales. Unse, Santiago del Estero.

MONTAÑA, C. 1992. The colonization of bare areas in two-phase mosaics of an arid ecosystem. J. Ecol. 80:315-327.

METCALFE, C \& L. CHALK. 1957. Anatomy of the dicotyledons. Leaves, stem, and wood in relation to taxonomy with notes on economic uses. Vol. II. Claredon Press, Oxford.

MITLÖHNER, R. 1990. Die konkurrenz der HolzagwãchseimregengrünenTrockenwald des Chaco Boreal, Paraguay. UniversitätGöttinger, GöttingerBeitr. Zur Land-und Forstwirtschaft in den Tropen und Subtropen. 54: 1-177.

MOSTACEDO, B., \& T. FREDERICKSEN. 2000. Manual de métodos básicos de muestreo y análisis en ecología vegetal. Proyecto de Manejo Forestal Sostenible (BOLFOR), Santa Cruz de la Sierra.

PIRE, E. F. \& LEWIS, J. P. 1993. Los Matorrales de Allenrolfea vaginata (Gris.) OK del Chaco Santafesino. Natura Neotropicalis 1 (24/25): 1-8.

PLANEAR. http://www.lista-planear.org/ [acceso: 21 de febrero del 2015]

QUERO J.L. 2006. SADIE como herramienta de cuantificación de la heterogeneidad espacial: casos prácticos en el Parque Nacional de Sierra Nevada (Granada, España). Ecosistemas 15 (3): 40-47.

RAGONESE, A. 1951. La Vegetación de la República Argentina. II.- Estudio fitosociológico de las Salinas Grandes. Revista Inv. Agric. 5: 1-233.

RAGONESE, A.E. \& J.C. CASTIGLIONI. 1970. La vegetación del parque chaqueño. Bol. Soc. Arg. Bot. 11 (supl.): 133-160.

SADIEShell v 2.0.C 2008. Kelvin F. Conrad and Joe N. Perry. http://home.cogeco.ca/ sadieexplained/index. html

SIGSE. Sistema de Información Geográfica de Santiago del Estero. http://sigse.inta.gov.ar/website/SigSE_2/ viewer.htm [acceso: 14 septiembre 2009] 
SURFER V 8.01. Surface Mapping System (C 1993-2002, Golden Software, Inc.

TIRADO, R. \& F I. PUGNAIRE. 2003. Shrub spatial aggregation and consequences for reproductive success. Community Ecology. Oecologia 136: 296301.

VELASCO MOLINA, H. 1983. Uso y manejo del suelo. Limusa, México.

VILLAGRA, P. E., C. GIORDANO, J. A. ALVAREZ, J. B. CAVAGNARO, A. GUEVARA, C. SARTOR \& S. GRECO. 2011. Ser planta en el desierto: estrategias de uso de agua y resistencia al estrés hídrico en el Monte Central de Argentina. Ecología austral 21: 29-42.
ZARLAVSKY, G. E. (ed.). 2014. Histología vegetal: técnicas simples y complejas. 1a ed. Sociedad Argentina de Botánica, Buenos Aires.

ZÚÑIGA, B., G. MALDA, \& H. SUZÁN. 2005. Interacciones Planta-Nodriza en Lophophora diffusa (Cactaceae) en un Desierto Subtropical de México. Biotropica 37: 351-356.

Recibido el 31 de octubre de 2014, aceptado el 25 de febrero de 2015. 
